\title{
Laparoscopic adrenalectomy for Conn syndrome
}

\author{
K.A.P.R. Kumarasinghe ${ }^{1}$, S.K. Kollure ${ }^{1}$, R. Amerasena ${ }^{2}$ \\ 1 Department of Surgery, National Hospital Sri Lanka. \\ 2 Department opf Anaesthesia, National Hospital Sri Lanka.
}

Keywords: Adrenalectomy, laparoscopy, Conn's adenoma

\section{Introduction}

Since its introduction in the 1990s, laparoscopic or retroperitoneoscopic adrenalectomy has become the 'gold standard' in the resection of adrenal tumours, except for tumours with signs of malignancy $[1,2]$. The view of the operative field obtained during a transperitoneal laparoscopic approach is superior to that obtained by open approach.

\section{Case Report}

A 36 year old male with an episode of severe headache and short term memory impairment was admitted to medical casualty. On admission his blood pressure was 260/170 mmHg with papilloedema. An urgent non contrast computerised tomograghy (NCCT) of brain revealed bleeding into choroid plexus (Figure 1). Neurosurgically he was managed conservatively. His blood pressure was controlled on losartan, nifedipine and prazocin.

Further investigations revealed hypokalaemia ( $2.6 \mu \mathrm{mol} / \mathrm{l})$ with elevated serum aldosterone levels $(20.1 \mathrm{ng} / \mathrm{dl})$. Serum renin level was markedly reduced $(0.29 \mathrm{ng} / \mathrm{ml} / \mathrm{hr})$. His urinary VMA level was normal (9.98). CT abdomen detected a lesion measuring $1 \times 1$ $\mathrm{cm}$, in the right adrenal gland which is consistent with an adenoma (Figure 2). Diagnosis of Conn's adenoma was made. The patient was referred to our surgical unit.

After preoperative evaluation of the patient, laparoscopic adrenalectomy was planned. Surgery was performed under general anaesthesia with invasive monitoring.

Patient was put on lateral decubitus position, four port

Correspondence: K.A.P.R. Kumarasinghe

E-mail: prabath14@gmail.com entry made into the peritoneal cavity (Figure 3 ).

Liver was retracted using a fan retractor. Lateral peritoneal reflection of the right triangular ligament was divided with ultrasonic shears. Liver retracted medially to provide exposure to right kidney and adrenal gland. Gerotas fascia over the midportion of the kidney opened, perinephric fat dissected over its superior pole. Lateral edge of the inferior vena cava (IVC) identified which formed the medial margin of the dissection. The adrenal gland was identified by its orange yellow colour [3]. Short adrenal vein identified and divided between haemostatic clips. Adrenal gland was gradually dissected and direct adrenal branch from aorta and branch from inferior phrenic artery divided with ultrasonic shears. IVC retracted medially and extension of the gland behind it dissected out.

Endobag extraction of specimen via a $5 \mathrm{~cm}$ incision done. There was no measurable blood loss and the

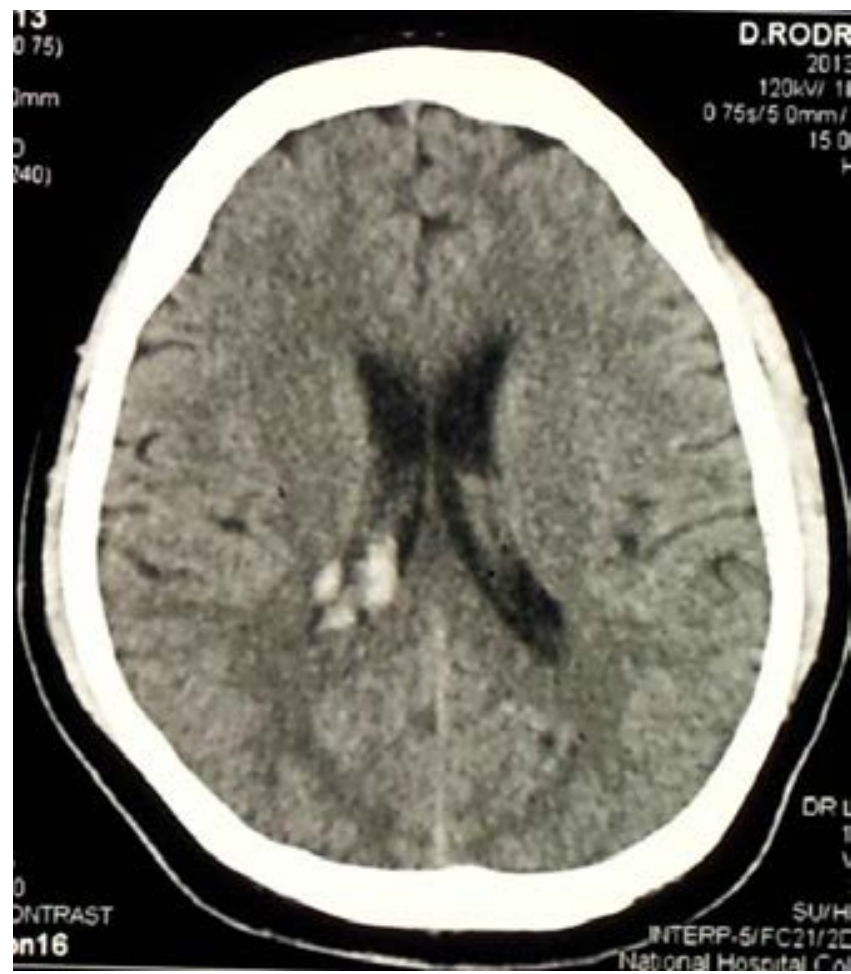

Figure 1. CT brain showing bleeding into choroid plexus. 


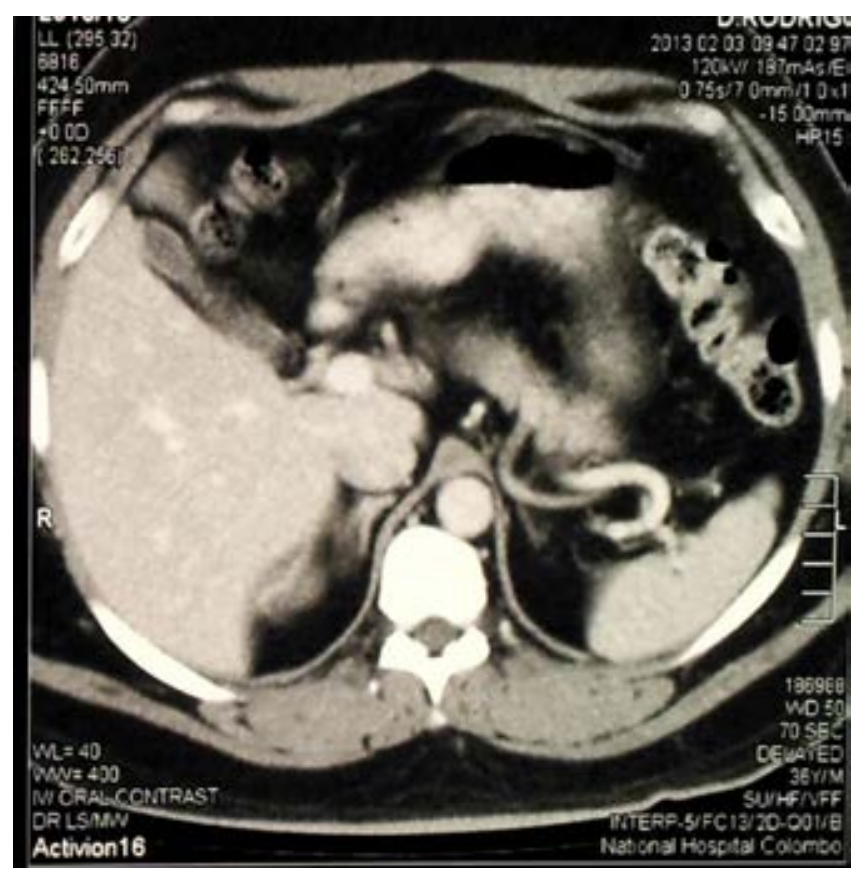

Figure 2. CT abdomen showing an adrenal adenoma on the right side

procedure took 80 minutes. Histology confirmed adrenal adenoma in right adrenal gland.

He was discharged on 4th post operative day. On discharge his blood pressure control was maintained only on losartan.

\section{Discussion}

In $80 \%$ of cases primary hyperaldosteronism is caused by an aldostrone secreting adenoma in one adrenal gland, a condition referred to as conn syndrome. In most cases adenomas are solitary and small $(<2 \mathrm{~cm}$ in diameter) [4].

Laparoscopic adrenalectomy is the commonest surgical approach currently in use. It is associated with reduced wound, chest and gastrointestinal complications, decreased hospital stay, shorter recovery time and improved patient satisfaction compared with open surgery [5].

Unilateral laparoscopic adrenalectomy is an effective therapy in patients with clear evidence of unilateral or asymmetrical bilateral disease. A subtotal resection is favoured in the case of a typical Conn's adenoma. In $10-30 \%$ of patients who undergo an adrenalectomy, hypertension persists despite adequate diagnostic

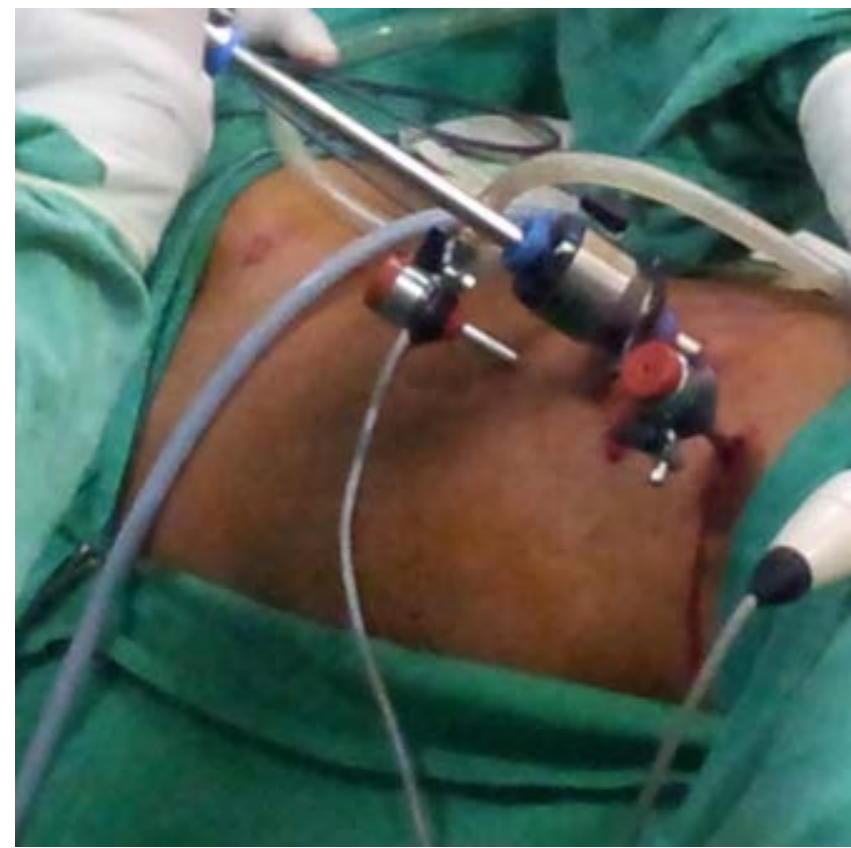

Figure 3. Port position

work-up and treatment [1].

Currently in Sri Lanka laparoscopic equipment and expertises are limited to few centres. Therefore open adrenalectomy is more commonly perfomed. With adequate facilities and surgical and anaesthetic expertises, laparoscopic adrenalectomy provides a revolutionary change over the conventional open method.

\section{References}

1. Norman S. Williams, Christopher J.K. Bulstrode, P. Ronan O'Connel: Baily and Love. 25th edition. Arnold; 2008

2. Matthew J. Mellon, Amanjot Sethi, Chandru P. Sundaram. Laparoscopic Adrenalectomy: Surgical techniques. Indian Journal of Urology. 2008; Oct-Dec24(4): 583-589

3. Carol E.H.Scott- Conner, David L. Dawson: Operative Anatomy. 3rd edition. Philadelphia: Lippincott Williams \& Wilkins; 2009

4. Vinay Kumar, Abdul K. Abbas, Nelson Fausto, Richard N, Mitchell. Robbins: Basic Pathology. 8th edition. Elsevier; 2009

5. Saba Balasubramanian, Barney Harrison. Investigation and management of adrenal disease. Surgery (Oxford). 2011; 459-464 


\section{Key points:}

- Laparoscopic adrenalectomy is the commonest surgical approach currently in use.

- Unilateral laparoscopic adrenalectomy is effective in patients with unilateral or asymmetrical bilateral disease.

- Subtotal resection is favoured in typical cases of Conn's disease. 